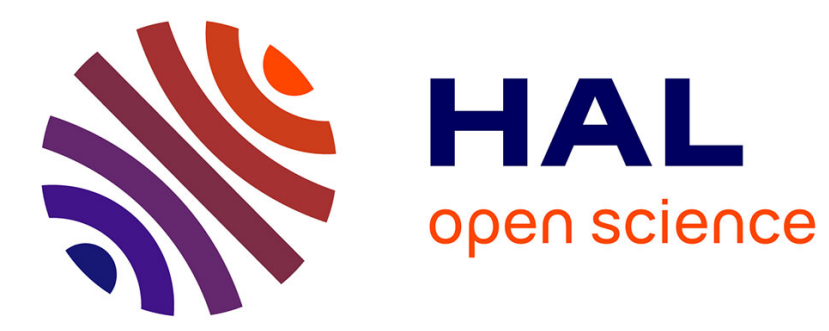

\title{
Le langage lyrique en Chine populaire: la modernité et l'après-Mao
}

Gregory B. Lee

\section{To cite this version:}

Gregory B. Lee. Le langage lyrique en Chine populaire: la modernité et l'après-Mao. Mots: les langages du politique, 2001, 66 (66), pp.125-133. 10.3406/mots.2001.2596 . halshs-02182704

\section{HAL Id: halshs-02182704 https://shs.hal.science/halshs-02182704}

Submitted on 13 Jul 2019

HAL is a multi-disciplinary open access archive for the deposit and dissemination of scientific research documents, whether they are published or not. The documents may come from teaching and research institutions in France or abroad, or from public or private research centers.
L'archive ouverte pluridisciplinaire HAL, est destinée au dépôt et à la diffusion de documents scientifiques de niveau recherche, publiés ou non, émanant des établissements d'enseignement et de recherche français ou étrangers, des laboratoires publics ou privés. 
Lee, Gregory B. ......[IETT, Université de Lyon] Le langage lyrique en Chine populaire : la modernité et l'aprèsMao. In: Mots, $\mathrm{n}^{\circ} 66$, juillet 2001. Discours d'Asie : identités et ruptures, sous la direction de Patrick Beillevaire ISSN: $0243-6450$

E-ISSN: $1960-6001$

DOI: $10.3406 /$ mots. 2001.2596

https://www.persee.fr/doc/mots_0243-6450_2001_num_66_1_2596

Langage lyrique chinois: la modernité et l'après Mao

Langage lyrique chinois: la modernité et l'après Mao Cet article traite de la contribution du langage poétique à la création et au développement d'une langue chinoise moderne. Pendant le vingtième siècle ce langage a suivi un parcours interrompu à de nombreuses occasions par l'ingérence politique, et a finalement atteint à travers une transgression nécessaire de la syntaxe une puissance éloquente.

Mots clés : langue, poésie, modernité, syntaxe, Duoduo

The Language of Chinese Lyricism: Modernity and the Post-Mao Period This article discusses the contribution of the language of poetry to the creation and development of the modern Chinese language in general. Having encountered considerable political interference during the twentieth century, this language finally attained an eloquence and power born of a necessary syntactic transgression.

[L'histoire culturelle et intellectuelle chinoise du vingtième siècle peut être lue comme une histoire linéaire, parallèle aux efforts de la Chine pour compenser les effets du développement technologique et économique inégal si brutalement exposé par l'agression impérialiste japonaise et occidentale, mais avant tout britannique d"'ouverture" de la Chine. Ce serait un méta narratif nationaliste linéaire de plus de cent cinquante ans, interrompu à intervalles réguliers par des luttes internes et des guerres.

Dans cette même période des histoires intellectuelle et politique de la Chine, nous pouvons trouver l'histoire d'une tentative de rupture d'avec les 
Lee, Gregory B. ......[IETT, Université de Lyon] Le langage lyrique en Chine populaire : la modernité et l'après-

Mao. In: Mots, $\mathrm{n}^{\circ}$ 66, juillet 2001. Discours d'Asie : identités et ruptures, sous la direction de Patrick Beillevaire

ISSN: 0243-6450

E-ISSN: $1960-6001$

DOI: $10.3406 /$ mots.2001.2596

https://www.persee.fr/doc/mots_0243-6450_2001_num_66_1_2596

modes féodaux de pensée et de reproduction culturelle, et de tentatives simultanées de négociation d'un modèle de modernité imposé du dehors. Cela serait moins un modèle linéaire qu'une conceptualisation de cette période de temps comme d'un long moment, d'une longue durée. A l'intérieur d'un tel cadre, les problèmes culturels chinois ainsi que ceux de la modernité en général, fusionnent en une problématique générale du postcolonialisme.]

A partir de la fin du dix-neuvième siècle la classe intellectuelle chinoise ressentit le besoin de produire des textes utiles à la modernisation de la Chine. Les énormes différences entre la langue classique écrite et la langue moderne parlée excluaient la possibilité de populariser ou de démocratiser la première, et, qui plus est, les intellectuels qui constituaient l'avant-garde de ce mouvement trouvaient eux-mêmes difficile de se séparer de cette langue de la poésie, de la bureaucratie, la langue des examens impériaux, la langue enfin dans laquelle ils avaient reçu leur laborieuse formation.

La classe intellectuelle ressentait une obligation envers la nation : ils voyaient le besoin de moderniser la société, l'économie et les institutions de l'état, le besoin de se débarrasser de la domination des Manchous (la dynastie étrangère qui gouvernait la Chine depuis le dix-septième siècle), et 
Lee, Gregory B. ......[IETT, Université de Lyon] Le langage lyrique en Chine populaire : la modernité et l'après-

Mao. In: Mots, $\mathrm{n}^{\circ}$ 66, juillet 2001. Discours d'Asie : identités et ruptures, sous la direction de Patrick Beillevaire

ISSN: 0243-6450

E-ISSN: $1960-6001$

DOI: $10.3406 /$ mots. 2001.2596

https://www.persee.fr/doc/mots_0243-6450_2001_num_66_1_2596

le besoin de chasser les puissances occidentales. Ils étaient bien sûr confrontés à la contradiction classique, à la nécessité d'imiter l'Occident pour se défendre contre lui.

Le problème, en ce qui concernait la langue dans cette logique quasiment obligatoire de modernisation, était de savoir comment la réformer pour instruire le peuple, c'est-à-dire comment la démocratiser et en faire un instrument propre à décrire le monde moderne.

Une langue vernaculaire écrite existait depuis mille ans, mais cette langue négligée ne savait pas parler de la modernité. Tout simplement le vocabulaire n'existait pas. Aussi, pour traduire les idées et les objets de la modernité occidentale, il fallait soit créer de nouveaux mots basés sur le sens, soit translittérer, soit encore adopter les mots que les Japonais avaient déjà inventés à partir des caractères chinois. Il s'agissait de reconstruire cette langue, plutôt que d'en inventer une nouvelle, de la faire accepter pour qu'on l'utilise comme une langue de communication sophistiquée, et finalement, de la faire valoriser auprès de la classe intellectuelle et de la nouvelle bourgeoisie chinoise qui allaient construire la nouvelle Chine.

Quand la Chine est entrée au 20e siècle, la poésie était toujours le genre littéraire dominant, et en dépit des efforts de développement du 
Lee, Gregory B. ......[IETT, Université de Lyon] Le langage lyrique en Chine populaire : la modernité et l'après-

Mao. In: Mots, $\mathrm{n}^{\circ}$ 66, juillet 2001. Discours d'Asie : identités et ruptures, sous la direction de Patrick Beillevaire

ISSN: 0243-6450

E-ISSN: $1960-6001$

DOI: $10.3406 /$ mots. 2001.2596

https://www.persee.fr/doc/mots_0243-6450_2001_num_66_1_2596

roman, on croyait que pour implanter la langue vernaculaire dans une

Chine toujours confucéenne et conservatrice, il fallait démontrer qu'une poésie moderne était possible. Cela semblait au moins être l'attitude des intellectuels qui préconisaient les réformes politiques tels que Liang Qichao, Tan Sitong, Xia Zengyou. En 1896, ces derniers lancèrent une révolution qu'ils appelèrent « la révolution de la poésie » shijie geming.

Cette révolution en fait n'a produit qu'un très faible relâchement des contraintes de la poésie classique. Par contre, elle a signalé la volonté de rétablir une poésie qui soit capable de répondre à la réalité contemporaine.

A peu près à la même époque, le poète Huang Zunxian que beaucoup considèrent comme le précurseur du mouvement de poésie moderne chinoise, lança le slogan : « Ma main écrit ce que dit ma bouche, comment l'antiquité peut-elle me retenir ?» Cette phrase célèbre, souvent citée comme celle qui aurait annoncé la rupture préliminaire révolutionnaire d'avec la tradition poétique, en dit plus sur les bonnes intentions de son auteur que sur sa capacité à élaborer avec succès un style poétique malléable qui réponde à la réalité sociale et de le faire dans un langage compréhensible de tous. Ce n'est que quand on lit la phrase en chinois, que son échec au niveau linguistique saute aux yeux, tout en démontrant la 
Lee, Gregory B. ......[IETT, Université de Lyon] Le langage lyrique en Chine populaire : la modernité et l'après-

Mao. In: Mots, $\mathrm{n}^{\circ}$ 66, juillet 2001. Discours d'Asie : identités et ruptures, sous la direction de Patrick Beillevaire

ISSN: 0243-6450

E-ISSN: $1960-6001$

DOI: $10.3406 /$ mots. 2001.2596

https://www.persee.fr/doc/mots_0243-6450_2001_num_66_1_2596

difficulté rencontrée par les poètes dans leurs efforts de se détacher de la langue et des règles classiques. Parce que la phrase est en fait écrite en chinois classique. Huang écrivait donc toujours en chinois ancien et sa prose autant que sa poésie était toujours remplie d'allusions aux classiques et à la poésie qui remontaient à deux ou trois mille ans. La seule sphère dans laquelle il se démarquait de la tradition était celle des thèmes qu'il abordait. Huang écrivait des poèmes qui traitaient de pays étrangers et des symboles de la modernité occidentale tels que la tour Eiffel. Mais tout cela était toujours enveloppé d'une langue poétique et d'un style qui rappelaient la poésie et les écrits d'antan.

Liang Qichao, le grand réformateur, considérait Huang comme le poète principal de la révolution poétique. Quant à sa poésie à lui, elle était pleine de notions contradictoires sur la réforme de la poésie, et il était clair qu'il n'était pas convaincu que la poésie devait être écrite dans une langue purement vernaculaire. Et, dans la poésie de Huang, les références à la modernité se heurtent aux expressions classiques et aux allusions conventionnelles. Par exemple, il existe deux poèmes sur les villes de Londres et de Paris. 
Lee, Gregory B. ......[IETT, Université de Lyon] Le langage lyrique en Chine populaire : la modernité et l'après-

Mao. In: Mots, $\mathrm{n}^{\circ}$ 66, juillet 2001. Discours d'Asie : identités et ruptures, sous la direction de Patrick Beillevaire

ISSN: 0243-6450

E-ISSN: $1960-6001$

DOI: $10.3406 /$ mots. 2001.2596

https://www.persee.fr/doc/mots_0243-6450_2001_num_66_1_2596

Dans le poème intitulé « Zai Lundun xie zhen zhi gan » (En écrivant un témoignage véridique à Londres) il y a beaucoup d'allusions à l'héritage littéraire classique : des phrases entières tirées de la poésie de Du Fu (Tou Fou), du Livre des Odes, le classique chinois de la poésie ainsi que de beaucoup d'autres textes canoniques. Ces emprunts étaient-ils intentionnels ? Est-ce que cette utilisation de ce qu'on peut considérer être des clichés prouve seulement que le poète ne faisait qu'écrire dans l'unique style qu'il pouvait concevoir, ou est-ce que l'emprise du style poétique classique de composition était si forte que le poète utilisait ce genre de phrases sans le savoir? La première interprétation semble la bonne, car le poète ne pourrait avoir employé sans s'en rendre compte le nombre d'allusions qui se trouvent dans ces poèmes. C'est néanmoins une indication de la difficulté de se débarrasser de la socialisation d'un système éducatif et linguistique sur lequel la vie culturelle et intellectuelle reposait; il ne faut pas oublier que jusqu'en 1905 toute éducation avait un but pratique, celui de la réussite des concours impériaux. De plus, le choc provoqué par cette poésie modernisée, même de manière superficielle, révèle la nature conservatrice du goût poétique contemporain, car, l'incorporation de thèmes et de noms de lieux étrangers, sans même toucher à la métrique 
Lee, Gregory B. ......[IETT, Université de Lyon] Le langage lyrique en Chine populaire : la modernité et l'après-

Mao. In: Mots, $\mathrm{n}^{\circ}$ 66, juillet 2001. Discours d'Asie : identités et ruptures, sous la direction de Patrick Beillevaire

ISSN: 0243-6450

E-ISSN: $1960-6001$

DOI: $10.3406 /$ mots.2001.2596

https://www.persee.fr/doc/mots_0243-6450_2001_num_66_1_2596

chinoise et sans renoncer à la composition allusive, était considérée comme un départ audacieux et moderne de la tradition.(1)

L'insatisfaction radicale face à la tradition classique avait commencé avec la prise de conscience que la Chine était impuissante face à l'agression colonialiste étrangère, en particulier celle des Britanniques. Cela avait été prouvé au dix-neuvième siècle par la défaite de la Chine lors des guerres de l'opium, mais l'insatisfaction et les appels renforcés à une rupture intellectuelle et créative totale avec le passé, n'ont atteint leur apogée qu'après l'échec apparent de la révolution chinoise de 1911 d'accomplir de réels changements, et surtout après le traitement honteux infligé à la Chine par le traité de Versailles de 1919 qui maintint les colonies européennes en Chine et transféra celles de l'Allemagne aux Japonais.

La nouvelle poésie allait donc devoir attendre une nouvelle génération, celle du 4 mai 1919. En dehors des quelques poètes qui se sont basés sur le romantisme anglais ou allemand, les poètes qui cherchaient de nouvelles dispositions pour développer l'expression poétique chinoise se sont concentrés surtout sur la tradition et technique françaises du vers libre. Les aspects techniques du vers libre attiraient la nouvelle génération de poètes qui voulaient se libérer de l'emprise de la versification stricte 
Lee, Gregory B. ......[IETT, Université de Lyon] Le langage lyrique en Chine populaire : la modernité et l'après-

Mao. In: Mots, $\mathrm{n}^{\circ}$ 66, juillet 2001. Discours d'Asie : identités et ruptures, sous la direction de Patrick Beillevaire

ISSN: 0243-6450

E-ISSN: 1960-6001

DOI: $10.3406 /$ mots. 2001.2596

https://www.persee.fr/doc/mots_0243-6450_2001_num_66_1_2596

classique. De plus, le vers libre facilitait l'usage du nouveau langage en devenir qui se basait sur le chinois parlé du nord.

Les écrivains qui s'intéressent au modernisme littéraire ont souvent remarqué que le modernisme rompait avec la tradition, et qu'en Chine, au début du siècle, cette volonté de rupture avec le passé était très forte dans une société où l'environnement culturel conservateur était beaucoup plus puissant et astreignant que dans les cultures occidentales en manque d'originalité.(2)

Le développement d'une langue nationale vulgaire en tant qu'outil quotidien fut assez rapide. En revanche celui de la langue littéraire était plus lent et tortueux. Même si les exigences politiques d'après la première guerre mondiale et la montée du nationalisme et du communisme en Chine ne demandaient qu'une instrumentalisation de la langue et de la littérature, l'acceptabilité esthétique de la nouvelle langue littéraire était plus difficile à établir, surtout dans le domaine de la poésie qui avait constitué pendant plus de deux mille ans le mode littéraire principal. A peine cette nouvelle langue poétique basée sur la poésie moderne occidentale, et en particulier celle de France, commençait-elle à s'affirmer pendant les années 1930 et 1940, que la guerre et la révolution freinèrent son dynamisme, et après 
Lee, Gregory B. ......[IETT, Université de Lyon] Le langage lyrique en Chine populaire : la modernité et l'après-

Mao. In: Mots, $\mathrm{n}^{\circ}$ 66, juillet 2001. Discours d'Asie : identités et ruptures, sous la direction de Patrick Beillevaire

ISSN: 0243-6450

E-ISSN: $1960-6001$

DOI: $10.3406 /$ mots. 2001.2596

https://www.persee.fr/doc/mots_0243-6450_2001_num_66_1_2596

l'établissement de la Chine populaire en 1949, les expériences modernistes en poésie prirent fin. Pendant la révolution culturelle de 1966 à 1976, la littérature fut soumise à un instrumentalisme total, et le langage fut entièrement transformé par le discours maoïste qui dominait non seulement la vie culturelle, mais aussi la vie quotidienne et les actions et pensées humaines.

La poésie produite sous Mao, en particulier pendant la révolution culturelle, était censée être l'expression du peuple. En fait, au lieu d'encourager le peuple à apprendre à faire de la littérature, les autorités sélectionnaient et formaient des ouvriers-écrivains modèles qui écrivaient des poèmes comme celui qui suit, à la gloire du parti, de la domination industrielle de la nature, et de la mécanisation. Ce poème de Li Genbao est intitulé « Le Phare »:

La ligne générale est un phare brillant, Qui nous éclaire bien dans notre progression.

Hier se portait encor le chapeau du lettré.

Aujourd'hui nul chapeau, on retrousse ses manches.

A côté des machines on écrit des poèmes ;

Et les poètes c'est nous. Nous les ouvriers. (3)

Ou encore une éloge des cheminées: 
Lee, Gregory B. ......[IETT, Université de Lyon] Le langage lyrique en Chine populaire : la modernité et l'après-

Mao. In: Mots, $\mathrm{n}^{\circ}$ 66, juillet 2001. Discours d'Asie : identités et ruptures, sous la direction de Patrick Beillevaire ISSN: 0243-6450

E-ISSN: $1960-6001$

DOI: $10.3406 /$ mots. 2001.2596

https://www.persee.fr/doc/mots_0243-6450_2001_num_66_1_2596

Hautes, hautes vers les nues blanches

Elles roulent des fumées noires.

Quel arbre aura jamais leur taille,

Quel bambou leur charme?

Elles ne sont que des grands pinceaux

Calligraphiant les beaux printemps de la patrie. (4)

Nulle invention linguistique ici, juste une langue mise au service de l’idéologie, poésie devenue « litanie civique » comme disait B. Péret. (5)

C'est dans ce contexte de servitude à la patrie révolutionnaire à laquelle la poésie était enchaînée, que la poésie non-officielle, surnommée par les autorités menglongshi «poésie obscure » ou "poésie floue », est née.

Pour les jeunes intellectuels, les gardes rouges issus de bonne famille, et déçus par l'idéologie maoïste, la poésie clandestine sous forme samizdat constituait le genre littéraire préféré pour combattre le discours totalisant du communisme chinois, ce qui plus tard s'appellerait le Mao wenti, ou mode maoïste. Tout comme avant l'arrivée au pouvoir des communistes, la poésie chinoise se retrouvait à ce moment dans le langage et la logique du modernisme occidental. 
Lee, Gregory B. ......[IETT, Université de Lyon] Le langage lyrique en Chine populaire : la modernité et l'aprèsMao. In: Mots, $\mathrm{n}^{\circ}$ 66, juillet 2001. Discours d'Asie : identités et ruptures, sous la direction de Patrick Beillevaire ISSN: 0243-6450

E-ISSN: $1960-6001$

DOI: $10.3406 /$ mots. 2001.2596

https://www.persee.fr/doc/mots_0243-6450_2001_num_66_1_2596

C'est avec la réinvention du langage que beaucoup de jeunes survivants de la révolution culturelle choisirent de négocier et de réenregistrer le passé, et aussi, plus positivement, d'imaginer l'avenir. Eprouvant le besoin de dénaturaliser et de remodeler la langue d'après la révolution culturelle, le poète Duoduo (1951- ) écrira :

Avec des outils refaçonnés refaçonnons du langage

Avec du langage refaçonné

Continuons de refaçonner (6)

Si selon André Dhôtel la «seule manière de défendre la langue, c'est de l'attaquer » et si, de cette façon, « chaque écrivain est obligé de se faire sa langue ", il y a aussi un deuxième aspect de la littérature qui est l'invention d'une nouvelle langue dans la langue, par création de syntaxe. (7) On peut dire que le devenir de la langue se trouve dans la création syntaxique.

La nature de la langue chinoise veut que la création de mots ne puisse se faire qu'en juxtaposant des caractères existants (chaque syllabe ayant une valeur phonique monosyllabique) pour créer de nouveaux mots polysyllabiques. On ne crée que rarement des caractères neufs, et quoiqu'il en soit comme l'on dispose approximativement de 50,000 caractères en chinois, ce n'est guère nécessaire. (8)

Ainsi, c'est vraiment au niveau de la syntaxe et de l'innovation métaphorique que le poète chinois va pouvoir être inventif, en poussant toujours plus loin le sens des mots, et les possibilités d'ambiguïté à leurs 
Lee, Gregory B. ......[IETT, Université de Lyon] Le langage lyrique en Chine populaire : la modernité et l'après-

Mao. In: Mots, $\mathrm{n}^{\circ}$ 66, juillet 2001. Discours d'Asie : identités et ruptures, sous la direction de Patrick Beillevaire

ISSN: 0243-6450

E-ISSN: $1960-6001$

DOI: $10.3406 /$ mots. 2001.2596

https://www.persee.fr/doc/mots_0243-6450_2001_num_66_1_2596

limites, en déployant des mots dans des combinaisons impensables dans le langage quotidien et dans la langue officielle.

Cette création syntaxique a été l'essence de la poésie contemporaine chinoise non-officielle. Dans plusieurs des poèmes de Duoduo, la question de la langue est centrale. Un des ces poèmes d'après -exil, « Mei you » «Il n’y a pas », par exemple, tisse une négativité répétée, rythmique et pessimiste, un silence dans lequel la communication est absente. Un espace poétique qui n'a pas de limites.

Sauf le langage, qui fait face à la terre et sa frontière perdue

Sauf ma fenêtre, qui donne sur mon langage qui n'est plus compréhensible

Mais le langage qui résiste à la compréhension devient rapidement tout à fait absent :

Il n'y a pas de langage

Il n'y a que la lumière qui grince, grince de manière répétitive répétitive cette scie manœuvrée à l'aube (9)

Le poème raconte un processus, et le poème lui-même en fait partie. Le langage raconte sa propre disparition, pour se voir recréé dans la narration de sa propre destruction.

T. Adorno se demandait comment des gens pouvaient penser à faire de la poésie après Auschwitz, après les camps de concentration. On peut 
Lee, Gregory B. ......[IETT, Université de Lyon] Le langage lyrique en Chine populaire : la modernité et l'après-

Mao. In: Mots, $\mathrm{n}^{\circ}$ 66, juillet 2001. Discours d'Asie : identités et ruptures, sous la direction de Patrick Beillevaire

ISSN: 0243-6450

E-ISSN: $1960-6001$

DOI: $10.3406 /$ mots.2001.2596

https://www.persee.fr/doc/mots_0243-6450_2001_num_66_1_2596

aussi se demander comment après la destruction, la déception et la mort de dizaines de millions de Chinois pendant les années 1950 et 1960, il était possible de penser à écrire la poésie de nouveau. Mais, peut-être que seules la dénaturalisation de la langue et la réinvention d'un langage poétique peuvent après de tels moments historiques fournir les moyens de négocier les sentiments, le moyen de réorienter l'esprit vers la logique et la santé mentale, de remplir les ressources de l'espoir, et de nous aider à construire les matrices qui sont nécessaires aux changements. C'est dans le langage de la poésie que de tels changements peuvent être imaginés.

Certains aspects linguistiques particulier au chinois aident le poète qui cherche à réinventer et à dénaturaliser sa langue. Ainsi, en en exploitant ces possibilités, il parvient à investir son poème d'ambiguïté, de double sens, de tournures de phrase qui vont faire penser le lecteur, qui vont le pousser à penser autrement sa propre langue. Cette ambiguïté est renforcée par le fait que le chinois n'exige pas l'usage d'un pronom personnel avant le verbe (comme l'espagnol) et qu'il n'y a pas d'indication de la personne du verbe contenue dans le verbe même (à l'opposé de l'espagnol), car le verbe chinois ne se conjugue pas et ne donne pas d'indication du temps, tout comme le nom chinois ne se décline pas et n'a pas de genre. Tous ces 
Lee, Gregory B. ......[IETT, Université de Lyon] Le langage lyrique en Chine populaire : la modernité et l'après-

Mao. In: Mots, $\mathrm{n}^{\circ}$ 66, juillet 2001. Discours d'Asie : identités et ruptures, sous la direction de Patrick Beillevaire

ISSN: 0243-6450

E-ISSN: 1960-6001

DOI: $10.3406 /$ mots. 2001.2596

https://www.persee.fr/doc/mots_0243-6450_2001_num_66_1_2596

aspects de la langue chinoise accumulent les possibilités d'ambiguité. Un manque de «précision » qui va peut-être perturber le scientifique mais pas le poète. Il faut souligner que cette ambiguïté voulue et intentionnelle n'a rien à voir avec l'ambiguïté de la poésie chinoise classique dont l'opacité dépend d'une connaissance de nombreuses allusions littéraires et du minimalisme grammatical de la langue classique.

Dans le poème « Jours de fête » on trouve la strophe suivante :

Une femme à quatre membres recouverte d'hippocampes apparaît en flottant, immédiatement,

se dissout comme un comprimé, passe, par ton corps retenu il crée mon souvenir : Moi et toi, devenus deux blocs de pierre, s'embrassant sur le linteau d'un grand opéra (10)

Le poète emploie très peu de ponctuation et il n'est pas toujours clair qu'un vers enjambe le suivant ou non. Ici, est-ce que le mot «immédiatement » réfère à l'action précédente ou à celle qui suit ou encore aux deux ? Dans « il crée mon souvenir », le caractère traduit par il, $t a$, est neutre en chinois et donc ne peut désigner une personne, mais cela dit à quoi réfère-t-il, au corps, au comprimé ou à la scène toute entière ?

Pourtant, il ne s'agit pas d'images aléatoires, il s'agit d'une difficulté intentionnelle qui tente de trouver de nouvelles possibilités dans cette 
Lee, Gregory B. ......[IETT, Université de Lyon] Le langage lyrique en Chine populaire : la modernité et l'après-

Mao. In: Mots, $\mathrm{n}^{\circ}$ 66, juillet 2001. Discours d'Asie : identités et ruptures, sous la direction de Patrick Beillevaire

ISSN: 0243-6450

E-ISSN: $1960-6001$

DOI: $10.3406 /$ mots.2001.2596

https://www.persee.fr/doc/mots_0243-6450_2001_num_66_1_2596

langue qui était devenue si stérile et si pleine de sens excluant tout autre sens par le discours idéologique du régime de Mao. Le langage du poète d'après Mao doit être refaçonné, les mots placés dans des combinaisons et des situations rares et nouvelles. Cette poésie, déjà difficile à traduire en anglais, est pratiquement impossible à traduire en français si l'on veut préserver l'ambiguïté de la syntaxe. Afin d'illustrer la nature de ce problème il serait peut-être utile de donner en exemple un poème de Duoduo intitulé «Zai mudi»(Au cimetière), en transcription phonétique (pinyin) suivi de sa traduction littérale:

Zai mudi, er meiyou huiyi you hanxi, danshi bei tuichi mengzhe lian, wei xia qu

chang :

mei ren yao women, women zai yiqi shi women beihou de yun, yao women kao zai yiqi women beihou de shu, bici kao de geng jin

chang

yinwei shouru

xue cong tianshang lai, yinwei zhufu

feng zai cidi, cidi bian shi yiwang yue shi yuan li maidi, bian yue shi gudu

shouting 
Lee, Gregory B. ......[IETT, Université de Lyon] Le langage lyrique en Chine populaire : la modernité et l'aprèsMao. In: Mots, $\mathrm{n}^{\circ}$ 66, juillet 2001. Discours d'Asie : identités et ruptures, sous la direction de Patrick Beillevaire ISSN: 0243-6450

E-ISSN: $1960-6001$

DOI: $10.3406 /$ mots.2001.2596

https://www.persee.fr/doc/mots_0243-6450_2001_num_66_1_2596

ranhou shouge, hanleng, cai bozhong

renshou, suoyi jingjiu

xiangxin, yushi duchu :

you

you yige feixiang de jia - zai zhao women

dans le cimetière, mais/et il n'y a pas de souvenir il y a soupir(s), mais rapporté(s)

couvrant visage(s)/visage(s) couvert(s), se met/ se mettent/ se mettant à genoux

chante/chantent/chantant :

personne ne veut de nous, nous sommes ensembles

ce sont les nuages derrière nous, veulent que nous nous serrions

les arbres derrière nous, l'un l'autre se serrent encore proche

chante/chantent/chantant

Parce que humilié(e)(s)

neige vient du ciel, parce que bénédiction/béni(e)(s)

le vent (est) dans cet endroit, cet endroit est donc oubli/oublier/oubliant

plus est loin du champ de blé, plus est solitaire

écoute/écoutent/écoutant

et puis récolte(ent), glace froid, alors sème/sèment

souffrant/souffre(ent), donc perdurant/perdure(nt)

croyant/croit/croient/, puis récite(nt)/récitant

il y a/il a/ils ont 
Lee, Gregory B. ......[IETT, Université de Lyon] Le langage lyrique en Chine populaire : la modernité et l'après-

Mao. In: Mots, $\mathrm{n}^{\circ}$ 66, juillet 2001. Discours d'Asie : identités et ruptures, sous la direction de Patrick Beillevaire ISSN: 0243-6450

E-ISSN: $1960-6001$

DOI: $10.3406 /$ mots. 2001.2596

https://www.persee.fr/doc/mots_0243-6450_2001_num_66_1_2596

Il y a/il a/ils ont un/des foyer(s)/maison(s) qui plane(nt) ---qui nous cherche(nt).

Pour faire du sens d'un tel poème il faut employer la stratégie de lecture verticale dont parle Bachelard, c'est-à-dire que pour comprendre il faut remonter et redescendre le poème. En lisant la première strophe il y a plusieurs ambiguïtés. Il y a un manque total de pronoms personnels, une absence d'indication du nombre. Ainsi, il n'est pas clair s'il s'agit d'un ou de plusieurs soupirs, qui se couvre(nt) le visage, qui se met(tent) à genoux. Peut-être est-ce la voix du poème ? Et puis, qui chante ?

La deuxième strophe semble donner des indices. Il y a un pronom, women « nous » employé cinq fois en chinois. Mais ce «nous » réfère à qui ? Il s'agit cette fois sûrement, d'une voix plurielle du poème. Mais, supposons qu'il s'agisse des « soupirs » de la première strophe ? Il y a un autre indice presque imperceptible : le mot chang « chante(ent)» intercalé entre les deux premières strophes est suivi de deux points. C'est le sujet de la première strophe qui chante donc, et le contenu de cette strophe est du discours direct. Il faut quand même trancher. Est-ce que le sujet pluriel de 
Lee, Gregory B. ......[IETT, Université de Lyon] Le langage lyrique en Chine populaire : la modernité et l'après-

Mao. In: Mots, $\mathrm{n}^{\circ}$ 66, juillet 2001. Discours d'Asie : identités et ruptures, sous la direction de Patrick Beillevaire

ISSN: 0243-6450

E-ISSN: $1960-6001$

DOI: $10.3406 /$ mots. 2001.2596

https://www.persee.fr/doc/mots_0243-6450_2001_num_66_1_2596

la première strophe est un vague «nous »: des gens, des morts ? Ou est-ce vraiment les soupirs qui chantent?

Le mot chang est intercalé une deuxième fois, entre la deuxième et la troisième strophe, mais cette fois sans être suivi de deux points, et la strophe n'a pas de pronom. Est-ce que chang veut simplement clore ce discours direct et faut-il donc le traduire par le participe présent « chantant »?

Dans la troisième strophe qui ou quoi est « humilié »? Est-ce que le sujet est encore la voix du poème, ou un sujet non déclaré ? Ou est-ce « la neige » qui est « humiliée»? La syntaxe permet cette lecture. Ou est-ce encore que « la neige vient du ciel » parce qu'elle est « bénie » ou est-ce le vent qui l'est ? De plus, s'agit-il toujours des «soupirs » ou d'un sujet nébuleux ? Et qui ou quoi est solitaire?

Intercalé entre la troisième et la quatrième strophes, on trouve le mot shouting, « écouter, être à l'écoute», ou plus littéralement « recevoirécouter 》 dont le caractère shou est répété dans le premier vers de la quatrième strophe : Ranhou shouge... «Et puis après récolte(nt)...» où shouge plus littéralement veut dire «recevoir-couper». Ici, il s'agit toujours de l'impossibilité de préciser le sujet : « Nous souffrons » ou «il(s) 
Lee, Gregory B. ......[IETT, Université de Lyon] Le langage lyrique en Chine populaire : la modernité et l'après-

Mao. In: Mots, $\mathrm{n}^{\circ}$ 66, juillet 2001. Discours d'Asie : identités et ruptures, sous la direction de Patrick Beillevaire

ISSN: 0243-6450

E-ISSN: $1960-6001$

DOI: $10.3406 /$ mots. 2001.2596

https://www.persee.fr/doc/mots_0243-6450_2001_num_66_1_2596

souffre(nt)»? «Nous croyons» ou « il(s) croit/croient»? Et puis la strophe se termine avec deux points, ce qui nous indique encore qu'il s'agit de nouveau d'un discours direct dont le sujet «nous » est révélé par la suite.

Toute la question de ce poème réside dans l'ambiguïté du sujet. En lisant le poème en chinois on peut garder ses sens multiples, en le traduisant en français, encore plus qu'en anglais où l'on peut préserver un peu d'ambiguïté en déployant le gérondif, la langue nous pousse à préciser un sujet, à produire une lecture close. Bien sûr, une traduction facilement lisible est faisable, mais c'est à cette lecture fermée que le traducteur doit essayer de manière boiteuse de résister en laissant ouvert un maximum de sens :

Au cimetière, mais il n'y a pas de souvenir il y a des soupirs, mais reportés visages couverts, s'agenouillent

chantent :

personne ne veut de nous, nous sommes ensemble ce sont les nuages derrière nous, ils veulent que nous nous serrions

Les arbres derrière nous, se serrent encore plus

en chantant 
Lee, Gregory B. ......[IETT, Université de Lyon] Le langage lyrique en Chine populaire : la modernité et l'aprèsMao. In: Mots, $\mathrm{n}^{\circ}$ 66, juillet 2001. Discours d'Asie : identités et ruptures, sous la direction de Patrick Beillevaire ISSN: 0243-6450

E-ISSN: $1960-6001$

DOI: $10.3406 /$ mots. 2001.2596

https://www.persee.fr/doc/mots_0243-6450_2001_num_66_1_2596

à cause de l'humiliation

la neige vient du ciel, à cause de la bénédiction

il y a du vent ici, donc ici est l'oubli

plus c'est loin du champ de blé, plus c'est solitaire

en écoutant

et puis récolte, glacé, et seulement après semis

Souffrance, donc endurance

Croyance, et puis récitation :

il y a

il y a un foyer qui plane ---- qui nous recherche.

Selon Gaston Bachelard «la poésie refuse les préambules, les

principes, les méthodes, les preuves. Elle refuse le doute. Tout au plus a-telle besoin d'un prélude de silence. D'abord, en frappant sur des mots creux, elle fait taire la prose ou les fredons qui laisseraient dans l'âme du lecteur une continuité de pensée ou de murmure. Puis, après les sonorités vides, elle produit son instant. C'est pour construire un instant complexe, pour nouer sur cet instant des simultanéités nombreuses que le poète détruit la continuité simple du temps enchaîné. » (11)

La transgression de ce temps enchaîné est essentielle à la reconstruction du langage poétique chez le poète moderniste chinois à la fin du vingtième siècle. Un extrait d'un autre poème de Duoduo, «Une seule 
Lee, Gregory B. ......[IETT, Université de Lyon] Le langage lyrique en Chine populaire : la modernité et l'après-

Mao. In: Mots, $\mathrm{n}^{\circ}$ 66, juillet 2001. Discours d'Asie : identités et ruptures, sous la direction de Patrick Beillevaire

ISSN: 0243-6450

E-ISSN: $1960-6001$

DOI: $10.3406 /$ mots. 2001.2596

https://www.persee.fr/doc/mots_0243-6450_2001_num_66_1_2596

histoire raconte tout son passé » montre justement une telle tension temporelle entre l'attraction du temps narratif et la force d'ancrage du temps arrêté, entre les perçus non-réfléchis fournis par la succession rapide des images de la caméra cinématique, et la lente étude du cliché.

Quand il ouvre les fenêtres de son corps qui donnent surl'océan Et saute vers le bruit des milliers de couteaux qui s'entrechoquent

Une seule histoire raconte tout son passé

Quand toutes les langues se tendent vers ce bruit

Et ré-aspirent les milliers de couteaux qui font ce bruit

Tous les jours se faufileront dans une seule journée

Ainsi, chaque année aura un joûr de plus

L'histoire qui raconte tout son passé est une histoire écrite en langue cinématique. Bien qu'une seule histoire suffise à dire tout un passé, il n'y a pas de récit régulier, et toute notion de temps normal est suspendue dès le début du poème :

Tous les jours se faufileront dans une seule journée Ainsi, chaque année aura un jour de plus (12)

Le «moment stabilisé » de Bachelard, l'instant poétique, est réitéré sans cesse dans ce poème. Plus loin, dans ce même poème, afin de dégager du temps réel un instant poétique dans lequel le temps bouge verticalement, le poète déploie des métaphores corporelles, ainsi que de motion et de stase 
Lee, Gregory B. ......[IETT, Université de Lyon] Le langage lyrique en Chine populaire : la modernité et l'après-

Mao. In: Mots, $\mathrm{n}^{\circ}$ 66, juillet 2001. Discours d'Asie : identités et ruptures, sous la direction de Patrick Beillevaire

ISSN: 0243-6450

E-ISSN: $1960-6001$

DOI: $10.3406 /$ mots. 2001.2596

https://www.persee.fr/doc/mots_0243-6450_2001_num_66_1_2596

pour marquer l'arrêt physique du temps : on empêche le soleil de se lever complètement, sa «tête » est abaissée, et le matin est forcé à rester. Le temps n'arrive plus à avancer. La pendule est cassée et « le temps pourri au-delà du tic-tac de l'horloge.»P Puis, le temps réel revient alors que « chaque nuit je fixe mon télescope sur ce point/jusqu'au moment où le soleil se meurt. »

Dans ce récit d'une mort, une cérémonie de la mort d'une ou même de plusieurs personnes, le temps historique, «mille ans détournent les yeux ». Là encore, le temps est sans temps, et la mort qui marche avec le temps, n'est qu'un «battement de cœur supplémentaire », elle s'est «morcelée », elle n'est rien de plus qu'un « grain de sable », comme si sans temps réel il n'y avait pas de mort réelle. Mais après l'instant poétique, il y a le retour du temps réel, et après le temps travaillé qui a produit l'instant poétique, il y a une plus grande prise de conscience de la réalité de la mort et de son inévitabilité.

Le langage banalisé, le langage littéraire du réalisme et du romantisme révolutionnaires qu'héritaient les jeunes poètes au crépuscule de la révolution culturelle n'était point capable de parler de la mort. A la fin de la révolution culturelle on trouva des milliers de récits et de poèmes qui 
Lee, Gregory B. ......[IETT, Université de Lyon] Le langage lyrique en Chine populaire : la modernité et l'après-

Mao. In: Mots, $\mathrm{n}^{\circ}$ 66, juillet 2001. Discours d'Asie : identités et ruptures, sous la direction de Patrick Beillevaire

ISSN: 0243-6450

E-ISSN: $1960-6001$

DOI: $10.3406 /$ mots.2001.2596

https://www.persee.fr/doc/mots_0243-6450_2001_num_66_1_2596

voulaient parler des injustices, des horreurs, des morts, mais ils le faisaient avec le langage officiel uniformisé qui justement avait manqué de raconter cette réalité au moment où elle se déroulait, avec le langage du mode maoïste. Ce que ces gens bien intentionnés ne comprenaient pas c'était que la langue aussi avait été tuée. Pour faire face à la réalité historique, pour la raconter, il fallait que le langage poétique prenne ses distances avec « le réel », avec le mode littéraire réaliste, avec le temps linéaire.

Les poètes d'après Mao, et en particulier, le poète Duoduo, ont créé, refaçonné un nouveau langage poétique, un langage que les premiers poètes innovateurs à la fin du dix-neuvième siècle ne pouvaient imaginer, afin de faire face à ce réel. Ils l'ont fait en cassant la langue de la période du Maoïsme, ils avaient même commencé avant la fin de la révolution culturelle dans la clandestinité. Comme l'aurait dit Gilles Deleuze, il s'agissait de l'invention d'une sorte de langue étrangère « qui n'est pas une autre langue, ni un patois retrouvé, mais un devenir-autre de la langue, une minoration de cette langue majeure.» C'est avec cette «langue refaçonnée » que les poètes contemporains continuent à refaçonner.

1. MARTIN, Helmut, "A Transitional Concept of Chinese Literature 1897-1917 : Liang Ch'i-Chao on Poetry Reform, Historical Drama and the Political Novel », Oriens Extremus 20, 1973, 196-197 : «Les paquebots, 
Lee, Gregory B. ......[IETT, Université de Lyon] Le langage lyrique en Chine populaire : la modernité et l'aprèsMao. In: Mots, $\mathrm{n}^{\circ} 66$, juillet 2001. Discours d'Asie : identités et ruptures, sous la direction de Patrick Beillevaire ISSN: $0243-6450$

E-ISSN: $1960-6001$

DOI: $10.3406 /$ mots. 2001.2596

https://www.persee.fr/doc/mots_0243-6450_2001_num_66_1_2596

les trains et le télégraphe figurent [dans sa poésie]. Une nouvelle terminologie politique exaltant "la démocratie, la liberté et l'égalité" revient ici et là [et le lecteur est confronté à des poèmes] dans lesquels de nouveaux objets importés entrent en conflit avec des allusions conventionnelles $\gg$.

2. Voir à ce sujet ma discussion du modernisme dans LEE, Gregory, Dai Wangshu: The Life and Poetry of a Chinese Modernist, Hong Kong, Chinese University Press, 1989.

3. LOI, Michelle, trad. , Poètes du peuple chinois, Paris, Pierre Jean Oswald, 1969, p.15.

4. Ibid., p 16.

5. PERET, Benjamin, Le déshonneur des poètes, Paris, Mille et une nuits, 1996, p. 17.

6. DUODUO, Licheng : Duoduo shixuan, [s.l.], [s.d.] publication samizdat, p. 77.

7. DELEUZE, Gilles, Critique et clinique, Paris, Les Editions de Minuit, 1993, p. 16.

8. On crée des caractères pour traduire les nouveaux éléments chimiques. Par exemple, le caractère chinois pour l'élément chimique nobélium est nuò qui est formé du radical ou clé pour métal et de la partie phonétique tiré du caractère nuò signifiant « assentiment, acceptation ».

9. DUODUO, manuscrit.

10. DUODUO, manuscrit.

11. BACHELARD, Gaston. Le Droit de rêver, Paris, Presses Universitaires de France, 1970, p. 224.

12. DUODUO, Licheng, pp. 73-75.

13 . DELEUZE, op. cit. p. 16.

Gregory B. Lee

Professeur de chinois et co-directeur de l'équipe de recherche CRITIC, Université Jean Moulin-Lyon 3

6 Cours Albert Thomas

69008 LYON

0492766604

gblee@karatel.fr 
Lee, Gregory B. ......[IETT, Université de Lyon] Le langage lyrique en Chine populaire : la modernité et l'aprèsMao. In: Mots, $\mathrm{n}^{\circ} 66$, juillet 2001. Discours d'Asie : identités et ruptures, sous la direction de Patrick Beillevaire ISSN: $0243-6450$

E-ISSN: 1960-6001

DOI: $10.3406 /$ mots. 2001.2596

https://www.persee.fr/doc/mots_0243-6450_2001_num_66_1_2596 